\title{
Multi-product Pricing Method Based on Improved Ant Colony Algorithm
}

\author{
Jing $\mathrm{Xu}{ }^{\mathrm{a},{ }^{*}, \text { Qiunan Meng }}$ \\ School of Dalian University of Technology, Dalian 116024, China \\ a718120609@qq.com
}

Keywords: Multi-product pricing, stochastic demand, product quality, improved ant colony algorithm

\begin{abstract}
Integrating with the due-date and quality demand of customers, a dynamic pricing model with stochastic demand and capacity constraint which aimed to maximize total profit and average quality was established for the multi-product pricing problem with random parameter. A weight space ant colony optimization algorithm (WSACO) was proposed, which allowed ant's search direction to adjust dynamically according to the current Pareto solutions, thus improving the global searching capability efficiently. Compared to the Bi-criterion Ant (BIANT) algorithm, it obtains higher solving accuracy, the results also show that the WSACO algorithm presents better performance in improving profit and product quality and provides method support for multi-product pricing decisions of enterprises.
\end{abstract}

\section{Introduction}

The traditional pricing methods often adopt cost-plus pricing method, without considering the influence of customer demand, therefore, they always lead to loss of business even if product prices are high. With the popularity of the make-to-order (MTO) model, customer demand fluctuates seriously, and they are more sensitive to price, due dates and product quality, which makes pricing more difficult. In the customer personalized dominated time, integrating with the due date and quality demand of customers, it is vital to formulate reasonable product pricing standards with considering uncertain demand and limited capacity constraints to expand the profitability of enterprises.

In terms of pricing research with demand and capacity constraints, Gilbert ${ }^{[1]}$ deals with the problem of jointly optimize production schedules and pricing decision under the assumption that demand is a function of price and seasonal. Bajwa et al ${ }^{[2]}$ apply outer approximation algorithm to solve the multiproduct pricing problem with dynamic demand and time-varying capacity constraints, the results show that the solution approach can obtain optimal prices in different demand scenarios of linear /nonlinear demand function. Deng et.al ${ }^{[3]}$ establish a joint production planning scheduling model under limited capacity and setup costs, and then they propose a product pricing model on the basis of planning scheduling model. The above studies research the product pricing problem with uncertain demand and capacity constraints, but fail to consider the influence of quality on pricing.

When it comes to joint pricing decisions, Li et.al ${ }^{[4]}$ study a joint lot-sizing and pricing problem that considers market factors, the traditional joint decision model is improved by adding stochastic demand and quantity constraints, and it is applicable to solve dynamic pricing problem of single product. Heuvel et.al ${ }^{[5]}$ develop a economic lot size model to synchronously optimize optimal price and order decision, with demand is deterministic and price-dependent and capacity is unlimited. They assume that price is definite and demand depends on price only, which makes the model too simple. Smith ${ }^{[6]}$ focus on a multi-product and multi-period lot-sizing and product pricing model, simulation experiment indicates that the proposed method can improve profit margin on different scales. The above articles mainly focus on joint optimization problems, but they ignore capacity influence.

Aiming at these problems, taking customer demand for due date and product quality into consideration under the MTO environment, a multi-product and multi-period dynamic pricing model is established with stochastic demand and capacity constraints, which intends to maximize total profit and average product quality. A WSACO (weight space ant colony optimization) algorithm is proposed, and it improves convergence rate and solution accuracy. All these ensure to pricing for products reasonably and improve the profitability of enterprises effectively. 


\section{Problem statement and formulation}

We consider the case of a MTO manufacturer who produces different products on a single machine without considering setup costs. There are $T(t=1,2, T)$ periods, and the firm sets up price and duedate intervals for every product at the beginning of each period. When customers arrive, they determine order quantity according to the evaluation of price, quoted due date and quality, therefore, we assume demand is a function of the three items. The firm develops production plan according to orders, production quantity of each product cannot exceed demand. Production capacity of the firm is fixed in each stage, but it can be improved by working overtime, outsourcing and so on.

Customers do not accept delivery in advance but delayed-sensitive. When the stochastic demand is realized, the manufacturer's production schedule may affect product's actual delivery time. If an order completes before the commitment due date, it occurs holding costs, in reverse, an order shipped after the commitment due date is considered late and incurs a tardiness penalty proportional to the time and the quantity delay. Shortages are allowed. Due to the initial investment in products is certain, we assume that the quality provided by the manufacturer is linear to the commitment due date ${ }^{[7]}$, in addition, upper limit exists because of the limited production capacity.

\subsection{Notation}

$P_{j t}: P_{j t}=\left\{p_{j t}{ }^{1},, p_{j t}{ }^{\mathrm{i}},, p_{j t}{ }^{\mathrm{n}_{j}}\right\}$, set of price can be selected by product $j$ in period $t$;

$D U_{j t}: D U_{j t}=\left\{d_{j t}{ }^{1},, d_{j t}{ }^{\mathrm{k}}, d_{j t}{ }^{{ }^{j}}\right\}$, set of commitment due-dates for product $j$ in period $t$;

$x_{j t}$ : Production quantity for product $j$ in period $t$;

$c_{j t}$ : Production cost per unit of product $j$ in period $t$;

$h_{j t}, r_{j t}$ : Holding cost and tardiness penalty per unit of product $j$ in period $t, r_{j t \geqslant} h_{j t}^{[8]}$;

$I_{j t}$ : Inventory holding time of production $j$ in period $t$;

$V_{j k t}$ : Delayed delivery time of product $j$ which selected due-date $d_{j t}{ }^{\mathrm{k}}$;

$\omega_{j}$ : Shortage penalty parameter of product $j$;

$K_{t}$ : Production capacity of the firm in period $t$;

$v_{j}$ : Production capacity consumed by unit product $j$;

$U_{j}$ : Total quantity of product $j$;

$D_{j i k t}$ : Demand for product $j$ corresponding to price $p_{j t}{ }^{\mathrm{i}}$ and due-date $d_{j t}{ }^{\mathrm{k}}$ in period $t$;

$m_{j t}$ : Quality of product $j$ in period $t$;

\subsection{Model formulation}

The multi-product and multi-period dynamic pricing model with stochastic demand and capacity constraints is formulated as follows:

(1) To maximize total profit:

$$
\begin{aligned}
\max \pi= & \sum_{j=1}^{J} \sum_{i=1}^{n_{j}} \sum_{t=1}^{T} P_{j t}^{i} x_{i j t}-\sum_{j=1}^{J} \sum_{i=1}^{n_{j}} \sum_{t=1}^{T} c_{j t} x_{i j t}-\sum_{j=1}^{J} \sum_{t=1}^{T} h_{j t} I_{j t}-\sum_{j=1}^{J} \sum_{k=1}^{l_{j}} \sum_{t=1}^{T} r_{j t} V_{j k t}- \\
& \sum_{j=1}^{J} w_{j}\left(\sum_{t=1}^{T} \sum_{i=1}^{n_{j}} \sum_{k=1}^{l_{j}}\left(A_{j}-B_{j} P_{j t}^{i}-C_{j} d_{j t}^{k}+E_{j} m_{j t}+\xi_{j}\right)-U_{j}\right)
\end{aligned}
$$

(2) To maximize average quality of all products:

$$
\max \left(\sum_{j=1}^{J} m_{j t} / J\right)
$$

Subject to:

$$
\begin{aligned}
& x_{i j t}-\sum_{k=1}^{l_{j}} D_{j i k t} \leq 0, \forall j \in J, i=1,2, \ldots, n_{j} \\
& \sum_{j=1}^{J} v_{j} x_{i j t}-K_{t} \leq 0 \\
& I_{j t} V_{j k t}=0, \forall j, \forall t \\
& U_{j}=\sum_{i=1}^{n_{j}} \sum_{t=1}^{T} x_{i j t} \\
& D_{i j k t}=A_{j}-B_{j} P_{j t}^{i}-C_{j} d_{j t}^{k}+E_{j} m_{j t}+\xi_{j}
\end{aligned}
$$




$$
\begin{aligned}
& m_{j t}\left(d_{j t}^{k}\right)=\left(d_{j t}^{k}\right)^{\delta} \\
& \delta \in\left[\delta_{\min }, \delta_{\max }\right] \\
& p_{j t}{ }^{\mathrm{i}}, I_{j t}, x_{i j t}, V_{j k t} \geq 0
\end{aligned}
$$

Constraint set (3) limits quantity of product j produced in each period by demand, constraint set (4) ensures the total quantity produced in a period may not exceed the production capacity. Constraint set (5) indicates that holding cost and tardiness penalty not exist in the same period. Constraint (6) represents the calculation formula for total quantity of product $j$, constraint (7) is the formula of demand, $A_{j}, B_{j}, C_{j}, E_{j}>0$, where $A_{j}, B_{j}, C_{j}$ and $E_{j}$ represent sensitivity to market scale, price, due-date and quality for product $j$, respectively, $\xi_{j}$ is a random variable with PDF (probability density function $\mathrm{f}($.$) and CDF (cumulative distribution function) \mathrm{F}\left(\right.$.), the vector set $\xi_{=}\left(\xi_{1}, \ldots, \xi_{j}, \xi_{n}\right)$ represents the random data vector for customer demand quantity. Constraint (8) calculates the quality of product $j$, which is relation to commitment due-date, constraint (9) shows quality effect parameter has interval limitations. Constraint (10) is the non-negativity constraint.

\section{Solution approach}

We propose a WSACO algorithm to solve the pricing model (Take the above case as an example, similarly for multi-objective problems).

\subsection{Space searching strategy}

The optimization steps are: (1) the solution space is divided evenly according to the number of objectives; (2) ants are allocated to subspaces and randomly generate weight direction vector; (3) Transfer probability calculation. Ants prefer to transfer to the node whose probability is maximum; (4) The ants allocated to the subspaces in the next iteration is adjusted dynamically based on the number of Pareto solutions in the corresponding subspace. The two-dimensional weight space is as Figure 1:

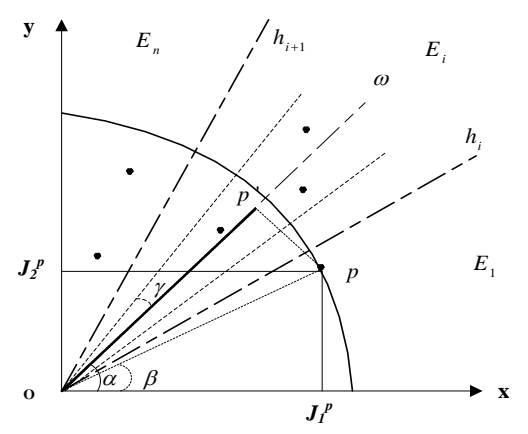

Fig. 1 Solution space division and weight vector decision

The two-dimensional coordinate system is divided into $n$ subspaces $\left(E_{k}(k=1,2, n)\right)$ by $n$ - 1 rays, direction vector $\omega$ generates randomly with the angle $\alpha$, starting from x-axis, and we set a small angle $\gamma$ beside $\omega$, thus, the interval $[\alpha-\gamma, \alpha+\gamma]$ is the transfer direction for ants. We suppose $\mathrm{p}$ is a outside point of $[\alpha-\gamma, \alpha+\gamma]$ with the angle $\beta$ from x-axis, $o p$ ' is the of $o p$, the weight of $o p$ ' along the $\mathrm{x}, \mathrm{y}$ axis is $\lambda_{1}=\frac{J_{1}^{p}}{\sqrt{1+\tan ^{2} \alpha}}, \lambda_{2}=\frac{J_{2}^{p} \cdot \tan \alpha}{\sqrt{1+\tan ^{2} \alpha}}$, so the weight of objective $J(J=1,2)$ along direction $\omega$ is $\lambda_{J}^{\prime}=\lambda_{J} / \sum_{J=1}^{2} \lambda_{J}$, therefore, ant's transition probability is calculated as follows:

$$
P_{s_{0} s}=\left\{\begin{array}{l}
\max _{s \in \Omega}\left(\prod_{J=1}^{2}\left(P_{s_{0} s}^{J}\right)^{\lambda_{j}}\right), q>q_{0} \\
\frac{\prod_{J=1}^{2}\left(P_{s_{0} s}^{J}\right)^{\lambda_{j}}}{\sum_{s^{\prime} \in \Omega} \prod_{J=1}^{2}\left(P_{s_{0} s}^{J}\right)^{\lambda_{j}}}, q \leq q_{0}
\end{array}\right.
$$

Where $q$ is a random parameter, $q \in(0,1)$. $Q 0$ is a threshold value, $P_{s o s}{ }^{J}$ is the probability of the objective $J(J=1,2)$, it is calculated by formula (12): 


$$
P_{s_{0} s}^{J}=\left\{\begin{array}{cc}
\frac{\left[\tau_{s_{0} s}^{J}\right]^{\alpha} \cdot\left[\eta_{s_{0}}^{J}\right]^{\beta}}{\sum_{s^{\prime} \in \Omega}\left[\tau_{s_{0} s}^{J}\right]^{\alpha} \cdot\left[\eta_{s_{0} s}^{J}\right]^{\beta}}, & , \text { else } \\
0 & =\Omega
\end{array}\right.
$$

The ants are allocated to subspaces equally in the initial iteration. The number of ants allocated to a subspace in the later iterations adjusts dynamically according to the distribution of Pareto set. The enter probability of ants is calculated as follows:

$z_{i}(N C)=\xi y_{i}(N C)+(1-\xi) z_{i}(N C-1)$

Where $N C$ is iterations, $\xi \in(0,1)$ is a weight coefficient, $y_{i}(N C)$ is calculated as:

$$
y_{i}(N C)= \begin{cases}\frac{1 /\left|f z p_{i}\right|}{\sum_{i=1}^{n} 1 /\left|f z p_{i}\right|} & \left|f z p_{i}\right| \neq 0 \\ \left|f z p_{i}\right| / \sum_{i=1}^{n}\left|f z p_{i}\right| & \text { else }\end{cases}
$$

\subsection{Parameters design of WSACO}

\subsubsection{Heuristic information calculation}

(1) Heuristic information for the first objective: We give the shorter due-date $\left(d_{j t}\right)$ a bigger heuristic information value.

$$
\eta_{s_{0} s}^{y_{1}}=1 / d_{j t}
$$

(2) Heuristic information for the second objective: The bigger are the quality effect parameters, the more heuristic information is given. ( $E_{j}$ is the sensitivity of demand to quality, ee is quality effect parameter relation to commitment due-dates.)

$$
\eta_{s_{0} s}^{y_{2}}=E_{j} \times e e_{j t}
$$

\subsubsection{Pheromone updating rules}

In order to obtain optimal solutions, the pheromone updating rule is designed as:

$$
\tau^{y_{i}}(N C+1)=(1-\phi) \tau^{y_{i}}(N C)+\sum_{a \in \mathbb{P}_{N C}} \Delta \tau^{a}(N C)
$$

Where $\Phi \in(0,1)$ is pheromone volatilization coefficient, $\mathrm{P}_{\mathrm{NC}}$ is the set that allows to update pheromone at iteration $N C, \Delta \tau^{\alpha}(N C)$ is the pheromone increment, which is calculated as ( $Q_{i}$ is adjustment coefficient for objective $f\left(y_{i}\right), i=1,2$.):

$$
\Delta \tau^{a}=Q_{i} / f\left(y_{i}\right)
$$

\subsection{Algorithm steps}

1: Initialize: Matrix and parameters setting, $m$ ants, maximum runtime is $t_{0}$;

2: Establish Pareto set $f z p$, ants are allocated to the subspaces equally;

3: Optimization direction $\alpha_{j}$ generates randomly for an ant in each subspace based on the strategy in 3.1 ;

4: Ants start to travel along the weight directions, transition probability to select the next node for objective $J$ is calculated by formulas (12), (15), (16).

5: Transition probability is normalized and direction values at all nodes are computed;

6: The nodes whose optimization range is in interval $[\alpha-\gamma, \alpha+\gamma]$ are recorded in the set $\Omega$, and the ants select the next node by formula (11);

7: The objective values are calculated by formulas (1), (2);

8: Calculate Pareto solutions in the current iteration and update $f_{z p}$, the path and direction of the Pareto solutions in $f z p$ are retained;

9: Formula (13) is used to compute the probability of ants in the next iteration and adjust the number of ants allocated to subspaces dynamically;

10: The path of the solutions in Pareto set can update their pheromone globally;

11: Record the run time $t$, if $t=t_{0}$, go to step 12, otherwise go to step3;

12: Output the Pareto set $f z p$. 


\section{Numerical study}

Garcia ${ }^{[12]}$ compared several improved ACO algorithms with genetic algorithms, BIANT algorithm presents better performance, so we apply it to compare with ours.

\subsection{Parameters setting}

There are 6 test cases (each contains 3 demand scenarios, represented by $S_{i}, i=1,2,3$ ) generate based on the literature ${ }^{[9]}$. Production quantity contains 3 levels $\left(J_{1}: 50, J_{2}: 100, J_{3}: 150\right)$, the corresponding due-dates are from discrete uniform distribution $([6,20],[15,20],[20,30]$, respectively), production capacity include 2levels $\left(C_{1}\right.$ : low capacity; $C_{2}$ : high capacity), prices are selected for each product from a bound set of integer values, $P(j)=\left\{p_{j}: 10 \leq p_{j} \leq 60\right\}, h_{j t}=0.5, r_{j t}=1, T=3$, the demand random variable $\xi_{j}$ obey an exponential distribution whose expectation is $\lambda, \lambda$ is from a uniform distribution, $[1,10] . J_{i} C_{j}(i=1,2,3 ; j=1,2)$ stands for each test case. $t=60 \mathrm{~s}, \alpha=0.01, \beta=0.2$, $\Phi=0.2, m=20, Q_{1}=1000, Q_{2}=0.0001, q_{0}=0$. The solution space is divided into 6subspaces, $\xi=0.2, N_{0}=6$, $q_{2}=0.8$. The weight for an ant $i$ is $w_{i}=(i-1) /(m-1)$ in BIANT algorithm.

\subsection{Algorithm results analysis}

WSACO algorithm implemented in MATLAB software helps to test the 6 cases, the maximize values solved by these two algorithms are in Table1.

Table 1 Objective Values comparison.

\begin{tabular}{ccclllll}
\hline \multirow{2}{*}{ Problem } & Demand & \multicolumn{3}{c}{ Obiective 1} & \multicolumn{3}{l}{ Obiective 2} \\
\cline { 2 - 7 } & scenario & $(1)$ & $(2)$ & $\%$ Imp & $(1)$ & $(2)$ & $\%$ Imp \\
\hline \multirow{3}{*}{$J_{1} C_{1}$} & $S_{1}$ & 15263 & 14538 & $5.0 \%$ & 0.9943 & 0.9544 & $4.2 \%$ \\
& $S_{2}$ & 16122 & 14238 & $13.2 \%$ & 0.9780 & 0.9153 & $6.9 \%$ \\
& $S_{3}$ & 15511 & 14295 & $8.5 \%$ & 0.9842 & 0.9554 & $3.0 \%$ \\
$J_{1} C_{2}$ & $S_{1}$ & 15426 & 15180 & $1.6 \%$ & 0.9980 & 0.9780 & $2.0 \%$ \\
& $S_{2}$ & 15248 & 14927 & $2.2 \%$ & 0.9967 & 0.8806 & $13.2 \%$ \\
& $S_{3}$ & 15388 & 14740 & $4.4 \%$ & 0.8910 & 0.8865 & $0.5 \%$ \\
$J_{2} C_{1}$ & $S_{1}$ & 21171 & 20222 & $4.7 \%$ & 0.9853 & 0.9435 & $4.4 \%$ \\
& $S_{2}$ & 20324 & 20212 & $0.6 \%$ & 0.9308 & 0.9070 & $2.6 \%$ \\
& $S_{3}$ & 20360 & 20166 & $1.0 \%$ & 0.9130 & 0.9105 & $0.3 \%$ \\
$J_{2} C_{2}$ & $S_{1}$ & 20008 & 19795 & $1.1 \%$ & 0.9752 & 0.9415 & $3.6 \%$ \\
& $S_{2}$ & 20293 & 18737 & $8.3 \%$ & 0.9402 & 0.9254 & $1.6 \%$ \\
& $S_{3}$ & 19930 & 19577 & $1.8 \%$ & 0.9896 & 0.9826 & $0.7 \%$ \\
$J_{3} C_{1}$ & $S_{1}$ & 22033 & 21179 & $4.0 \%$ & 0.9092 & 0.8936 & $1.7 \%$ \\
& $S_{2}$ & 22074 & 20272 & $8.9 \%$ & 0.9517 & 0.8939 & $6.5 \%$ \\
& $S_{3}$ & 22361 & 21932 & $2.0 \%$ & 0.9242 & 0.9155 & $1.0 \%$ \\
$J_{3} C_{2}$ & $S_{1}$ & 18503 & 17257 & $7.2 \%$ & 0.9239 & 0.8788 & $5.1 \%$ \\
& $S_{2}$ & 18362 & 17565 & $4.5 \%$ & 0.9650 & 0.8653 & $11.5 \%$ \\
& $S_{3}$ & 16547 & 15763 & $5.0 \%$ & 0.9117 & 0.9088 & $0.3 \%$ \\
\hline
\end{tabular}

Note: (1)=WSACO algorithm, (2)=BIANT algorithm, \%Imp=percentage improvement.

As Table 1 shows, the maximum values of the two objectives obtained by WSACO are better than the contrast algorithm in different tests and demand scenarios, and the statistical results indicate that the WSACO algorithm is more suitable for small and large scale problems. We select 3 tests $\left(J_{l} C_{2}\right.$, $J_{2} C_{1}, J_{3} C_{1} ; \mathrm{S}_{1}$ ) randomly and their results are shown in Fig.2.
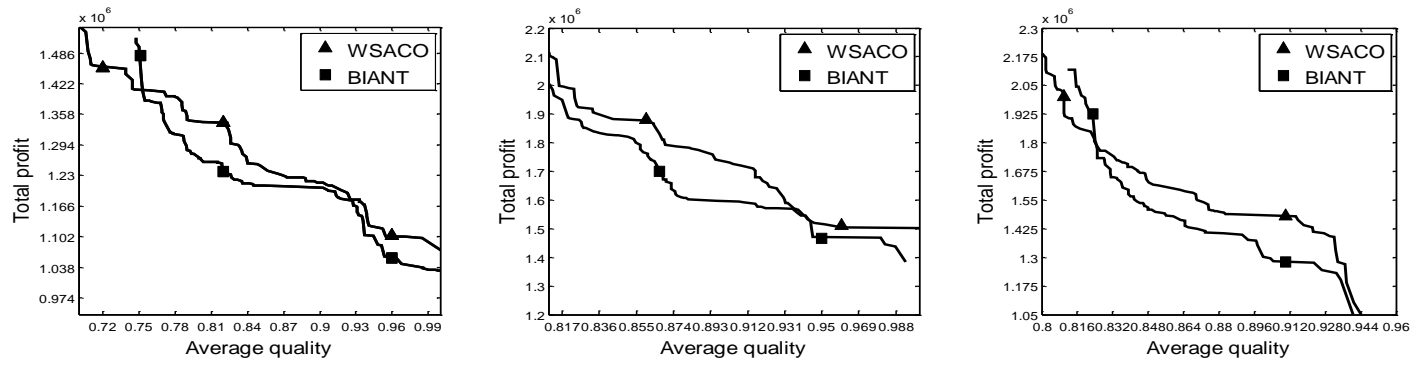

Fig. 2 Comparison of objective values

As Fig. 2 shows, in terms of maximum values, the WSACO algorithm finds better solutions; from the global point of view, the quality of solutions optimized by the WSACO algorithm is superior to the contrast algorithm.

We apply the evaluation indices in literature [10] to evaluate the performance of these two algorithms. The comparison results are shown in Table 2. 
Table 2 Comparison Results of indices.

\begin{tabular}{|c|c|c|c|c|c|c|c|c|c|}
\hline \multirow{2}{*}{ Problem } & \multirow{2}{*}{ Demand scenario } & \multicolumn{2}{|l|}{$\mathrm{D}_{\mathrm{av}}$} & \multicolumn{2}{|l|}{ TS } & \multicolumn{2}{|c|}{ ONVG } & \multicolumn{2}{|l|}{$\mathrm{C}$} \\
\hline & & (1) & (2) & (1) & (2) & (1) & (2) & (1) & (2) \\
\hline \multirow{3}{*}{$J_{1} C_{1}$} & $S_{1}$ & 0 & 14408.61 & 13.29 & 13 & 18 & 0 & 0 & 1 \\
\hline & $S_{2}$ & 0 & 6758.93 & 22 & 12.46 & 24 & 5 & 0 & 0.64 \\
\hline & $S_{3}$ & 23.74 & 26896.11 & 27.43 & 29.73 & 28 & 3 & 0.03 & 0.90 \\
\hline \multirow{4}{*}{$J_{1} C_{2}$} & $S_{1}$ & 0 & 13986.87 & 12.62 & 12.31 & 14 & 0 & 0 & 1 \\
\hline & $S_{2}$ & 1002.85 & 10527.66 & 21.14 & 19.71 & 19 & 8 & 0.14 & 0.64 \\
\hline & $S_{3}$ & 334.85 & 17813.66 & 25.08 & 26.08 & 23 & 12 & 0.15 & 0.56 \\
\hline & $S_{1}$ & 12185.54 & 14325.75 & 11.64 & 11 & 11 & 3 & 0.08 & 0.73 \\
\hline \multirow[t]{3}{*}{$J_{2} C_{1}$} & $S_{2}$ & 2611.07 & 13460.3 & 15.2 & 14.71 & 13 & 4 & 0.19 & 0.73 \\
\hline & $S_{3}$ & 0 & 23174.91 & 9.33 & 8.75 & 10 & 0 & 0 & 1 \\
\hline & $S_{1}$ & 6602.727 & 12990.15 & 13.54 & 13 & 8 & 6 & 0.43 & 0.54 \\
\hline \multirow[t]{3}{*}{$J_{2} C_{2}$} & $S_{2}$ & 0 & 33224.86 & 8 & 4 & 8 & 1 & 0 & 0.75 \\
\hline & $S_{3}$ & 427.03 & 9847.83 & 20.7 & 14.86 & 20 & 2 & 0.05 & 0.87 \\
\hline & $S_{1}$ & 0 & 39849.39 & 13.85 & 11.82 & 14 & 1 & 0 & 0.92 \\
\hline \multirow[t]{3}{*}{$J_{3} C_{1}$} & $S_{2}$ & 0 & 68352.91 & 9.78 & 8.5 & 10 & 1 & 0 & 0.89 \\
\hline & $S_{3}$ & 7346.96 & 114663.3 & 8.5 & 5.6 & 8 & 2 & 0.11 & 0.67 \\
\hline & $S_{1}$ & 4481.564 & 51008.63 & 12.67 & 10 & 11 & 4 & 0.15 & 0.6 \\
\hline \multirow[t]{2}{*}{$J_{3} C_{2}$} & $S_{2}$ & 5468.14 & 50715.25 & 7.71 & 6 & 7 & 3 & 0.125 & 0.5 \\
\hline & $S_{3}$ & 5600.11 & 11214.59 & 16.625 & 8 & 13 & 6 & 0.235 & 0.25 \\
\hline
\end{tabular}

Note: (1) = WSACO algorithm, (2) = BIANT algorithm.

Almost all the indicators of the WSACO algorithm are superior to BIANT algorithm, except the distribution indicator TS in $J_{l} C_{l}, J_{l} C_{2}$ (scenario 3) is less than the latter slightly. Distance indicator $\mathrm{D}_{\mathrm{av}}$ decrease $86.3 \%$ averagely, which indicates that the WSACO algorithm are more close to the Pareto front; the distribution indicator TS improve $26.8 \%$ averagely, which shows higher quality and more homogeneous distribution of the WSACO algorithm; the number of Pareto solutions ONVG increase $75 \%$ evenly; which represents a higher optimization capacity; Dominance indicator C decrease $81.4 \%$ evenly, it presents that the dominance degree of the Pareto set belongs to the WSACO algorithm is lower than the contrast algorithm and the WSACO algorithm obtains more precise solutions.

The above results show that the WSACO algorithm obtains higher profit and average quality, correspondingly, the proposed pricing method can obtain optimal price combination.

\section{Conclusion}

With the increasing competition of market, whether the pricing decision is reasonable or not determines the level of corporate profitability. Incorporating customer demand for due-date and quality, stochastic demand was simulated by a certain probability distribution. A multi-product dynamic pricing model with capacity constraint, whose objectives were to maximize total profit and average quality, was established, and it does improve the accuracy and reasonableness of product pricing. We proposed a WSACO algorithm based on weight space division and designed weight direction transfer mechanism for ants, the allocation strategy for ants was improved, which avoids premature convergence of the algorithm. Compared to the literature algorithm, the improved algorithm presented better solving accuracy, the results also showed that the proposed pricing method can optimize price effectively and improve profit and quality, which provides method support for corporate pricing decisions.

\section{References}

[1]. Stephen M. Gilbert, Coordination of pricing and multiple-period production across multiple constant priced goods, J, Management Science. 46 (2000) 1602-1616.

[2]. Naeem Bajwa, Charles R. Sox, Rafay Ishfaq, Coordinating pricing and production decisions for multiple products, J, Omega. 64 (2016) 86-101. 
[3]. Shiming Deng, Candace A. Yano, Joint production and pricing decisions with setup costs and capacity constraints, J, Management Science. 52 (2006) 741-756.

[4]. Hongyan Li, Anders Thorstenson, A multi-phase algorithm for a joint lot-sizing and pricing problem with stochastic demands, J, International Journal of Production Research. 52 (2014) 2345-2362.

[5]. Wilco Van Den Heuvel, Albert P.M. Watermans, A polynomial time algorithm for a deterministic joint pricing and inventory model, J, European Journal of Operational Research, 170 (2006) 463-480.

[6]. Neale R. Smith. A heuristic approach for a multi-product capacitated lot-sizing problem with pricing, J, International Journal of Production Research. 49 (2011) 1173-1196.

[7]. K. Palaka, S. Erlebacher, Dean H. Kropp, Lead-time setting, capacity utilization, and pricing decisions under lead-time dependent demand, J, IIE Transactions. 30 (1998) 151-163.

[8]. Andy Tsay, Narendra Agrawal, Channel conflict and coordination in the E-commerce age, J, Production \& Operations Management. 13 (2010) 93-110.

[9]. S. Kamal Chaharsooghi, Mahboobeh Honarvar, Mohammad Modarres et al, Developing a two stage stochastic programming model of the price and lead-time decision problem in the multiclass make-to-order firm, J, Computers \& Industrial Engineering. 61 (2011) 1086-1097.

[10]. Ling Wang, Bo Liu. Particle swarm optimization and scheduling algorithms, in Beijing, Tsinghua University Press, 2008, pp: 166-167. 